\title{
W Hya through the eye of Odin $\star$
}

\section{Satellite observations of circumstellar submillimetre $\mathrm{H}_{2} \mathrm{O}$ line emission}

\author{
K. Justtanont ${ }^{1}$, P. Bergman ${ }^{2}$, B. Larsson ${ }^{1}$, H. Olofsson ${ }^{1}$, F. L. Schöier ${ }^{1}$, U. Frisk ${ }^{3}$, T. Hasegawa ${ }^{4}$, Å Hjalmarson ${ }^{2}$,

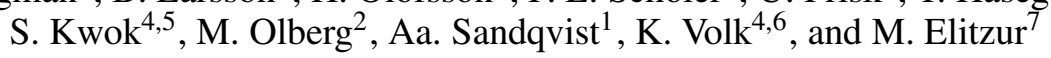 \\ 1 Stockholm Observatory, AlbaNova University Center, 10691 Stockholm, Sweden \\ e-mail: kay@astro.su.se \\ 2 Onsala Space Observatory, 43992 Onsala, Sweden \\ 3 Swedish Space Corporation, PO Box 4207, 17104 Solna, Sweden \\ 4 Dept. of Physics and Astronomy, University of Calgary, 500 University Drive NW, Calgary, Alberta T2N 1N4, Canada \\ 5 Institute of Astronomy and Astrophysics, Academia Sinica, PO Box 23-141, Taipei 106, Taiwan, ROC \\ ${ }^{6}$ Gemini Observatory, Southern Operation Center, La Serena, Chile \\ 7 Department of Physics and Astronomy, University of Kentucky, Lexington, KY 40506, USA
}

Received 2 March 2005 / Accepted 4 May 2005

\begin{abstract}
We present Odin observations of the AGB star W Hya in the ground-state transition of ortho- $\mathrm{H}_{2} \mathrm{O}, 1_{10}-1_{01}$, at $557 \mathrm{GHz}$. The line is clearly of circumstellar origin. Radiative transfer modelling of the water lines observed by Odin and ISO results in a mass-loss rate of $(2.5 \pm 0.5) \times 10^{-7} M_{\odot} \mathrm{yr}^{-1}$, and a circumstellar $\mathrm{H}_{2} \mathrm{O}$ abundance of $(2.0 \pm 1.0) \times 10^{-3}$. The inferred mass-loss rate is consistent with that obtained from modelling the circumstellar $\mathrm{CO}$ radio line emission, and also with that obtained from the dust emission modelling combined with a dynamical model for the outflow. The very high water abundance, higher than the cosmic oxygen abundance, can be explained by invoking an injection of excess water from evaporating icy bodies in the system. The required extra mass of water is quite small, on the order of $\sim 0.1 M_{\oplus}$.
\end{abstract}

Key words. stars: circumstellar matter - stars: evolution - stars: individual: W Hya - stars: late-type - stars: mass-loss stars: AGB and post-AGB

\section{Introduction}

One of the main characteristics of an Asymptotic Giant Branch (AGB) star is its cool, low-velocity outflow. In this outflow, molecules are formed according to the relative chemical abundance of carbon and oxygen (Olofsson 2003; Millar 2003, and the references therein). In addition to $\mathrm{H}_{2}, \mathrm{CO}$ is an abundant species irrespective of the $\mathrm{C} / \mathrm{O}$ ratio, but in $\mathrm{C} / \mathrm{O}<1$ (O-rich) objects its abundance is rivaled by that of $\mathrm{H}_{2} \mathrm{O}$, while in $\mathrm{C} / \mathrm{O}>1$ (C-rich) objects $\mathrm{HCN}$ and $\mathrm{C}_{2} \mathrm{H}_{2}$ also have sizable abundances. Likewise, there are dust signatures of amorphous carbon and $\mathrm{SiC}$ when $\mathrm{C} / \mathrm{O}$ is larger than unity, (Treffers \& Cohen 1974) while silicates are seen if the reverse is the case (Gilman 1969).

Early AGB stars tend to be semiregular variables with a low mass-loss rate, and these evolve into long-period Miratype variables where mass loss is more pronounced. The lifetime of a mass-losing AGB star is estimated to be about $10^{5} \mathrm{yr}$, and before evolving off the AGB the star undergoes an intense

* Based on observations with Odin, a Swedish-led satellite project funded jointly by the Swedish National Space Board (SNSB), the Canadian Space Agency (CSA), the National Technology Agency of Finland (Tekes), and Centre National d'Études Spatiales (CNES). The Swedish Space Corporation was the industrial prime contractor and is operating Odin. epoch of mass loss when most of the initial mass is lost, leaving behind a hot $\mathrm{C} / \mathrm{O}$ core which then ionizes the surrounding material resulting in a planetary nebula (see e.g., Iben \& Renzini 1993; Habing 1996).

In this paper we discuss circumstellar $\mathrm{H}_{2} \mathrm{O}$ observations of the well-studied O-rich semiregular variable W Hya, with a period of 382 days (Lebzelter et al. 2000). It has been detected in a number of circumstellar molecular line emissions, e.g., CO (Wannier \& Sahai 1986; Loup et al. 1993; Cernicharo et al. 1997; Kerschbuam \& Olofsson 1999), SiO maser (Pardo et al. 2004) and $\mathrm{OH}$ maser lines (Szymczak et al. 1998; Chapman et al. 1994; Etoka et al. 2003). It was also detected in water lines using the Infrared Space Observatory's (ISO) Short- and Long Wavelength Spectrometers (SWS and LWS) by Neufeld et al. (1996) and Barlow et al. (1996), respectively. Recently, the Submillimeter Wave Astronomy Satellite (SWAS) detected the ground-state ortho- $\mathrm{H}_{2} \mathrm{O}$ transition at $557 \mathrm{GHz}, 1_{10}-1_{01}$, as reported by Harwit \& Bergin (2002).

O-rich semiregular variable stars are seen to have multiple dust species condensing in their outflows (Cami 2002). The $10 \mu \mathrm{m}$ silicate feature, dominant in the spectra of Mira variables, may not be the strongest emission feature. This is clearly seen in the spectral energy distribution (SED) of W Hya. 
By fitting the SED with multiple dust species, Justtanont et al. (2004) derived a dust mass-loss rate of $3 \times 10^{-10} M_{\odot} \mathrm{yr}^{-1}$ (this is a lower limit as the $13 \mu \mathrm{m}$ dust feature was not fitted due to the lack of a suitable identification). This suggests that W Hya is a low-mass-loss-rate AGB star. However, its dust envelope is known to be very large, extending out to $40^{\prime}$ (Hawkins 1990).

\section{Observations}

The spectrum of the $\mathrm{H}_{2} \mathrm{O}\left(1_{10}-1_{01}\right)$ line at $557 \mathrm{GHz}$ $(\lambda=538.29 \mu \mathrm{m})$ was observed by the Odin satellite towards W Hya using the autocorrelation spectrometer (Nordh et al. 2003; Frisk et al. 2003). The resulting spectrum is a combination of two separate observing sessions, in December 2002 and July 2003, of a total of 185 orbits. As the positionswitching mode was used in these observations, the on-source integration time is $53.4 \mathrm{~h}$. The average system temperature for this frequency is $3200 \mathrm{~K}$. The calibration was done by switching between the internal hot load and the sky. The frequency resolution is $1 \mathrm{MHz}\left(=0.54 \mathrm{~km} \mathrm{~s}^{-1}\right.$ at $\left.557 \mathrm{GHz}\right)$. A sinusoidal baseline was subtracted and the data rebinned. Figure 1 shows the spectrum in terms of the observed antenna temperature, $T_{\mathrm{A}}$, which has not been corrected for the beam efficiency of $90 \%$.

The peak antenna temperature is $0.05 \pm 0.02 \mathrm{~K}$. The line flux is estimated to be $(3.6 \pm 1.2) \times 10^{-21} \mathrm{~W} \mathrm{~cm}^{-2}$, after correcting for the aperture efficiency of $70 \%$ (Frisk et al. 2003). The absolute uncertainty in the line flux is large as the data are noisy, and close to the line there are features which are the artefacts caused by the ends and the joining of two correlator bands. These are seen as "absorption features" at $V_{\mathrm{LSR}}$ of 4, 23 and $30 \mathrm{~km} \mathrm{~s}^{-1}$ (Fig. 1). The estimated uncertainty includes that due to the noise and the sinusoidal baseline subtraction, plus an absolute flux calibration uncertainty of $10 \%$. The latter is obtained by comparing the line fluxes of Odin and SWAS data for a number of sources (Hjalmarson et al. 2003). The line intensity is consistent with that obtained by SWAS after scaling the latter with the ratio of the Odin and SWAS beam sizes, 3.37 (Hjalmarson et al. 2003).

The centre velocity and the expansion velocity are estimated to be $42 \pm 1.5 \mathrm{~km} \mathrm{~s}^{-1}$ (LSR scale) and $7.0 \pm 1.0 \mathrm{~km} \mathrm{~s}^{-1}$, respectively. These values are consistent with the results from $\mathrm{CO}$ $J=1-0$ data (Kerschbaum \& Olofsson 1999), $J=2-1$ data (Cernicharo et al. 1997), $J=3-2$ and 4-3 data (Young 1995).

\section{Modelling of the molecular line emission}

In this section, we present our models for both the $\mathrm{H}_{2} \mathrm{O}$ and $\mathrm{CO}$ line emission from the circumstellar envelope of W Hya. The models used are based on the assumptions of a constant, isotropic mass-loss rate, and a constant expansion velocity, which result in a spherically symmetric gas outflow with an $r^{-2}$ density distribution. The mass-loss rate refers to the $\mathrm{H}_{2}$ loss rate which is the quantity used throughout this paper. This value needs to be multiplied by a factor of 1.4 to get the total gas mass-loss rate, due mainly to the presence of He.

In order to model the radiative transfer, we need to know the stellar luminosity. From the spectral energy distribution (SED),

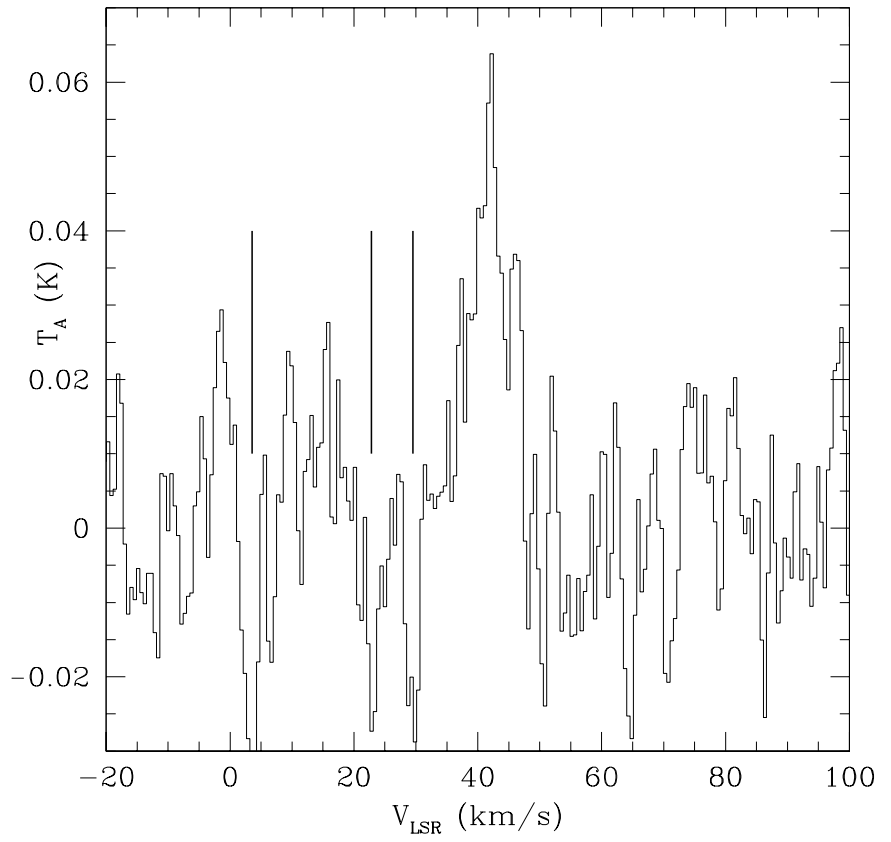

Fig. 1. The ground-state transition of ortho- $\mathrm{H}_{2} \mathrm{O}, 1_{10}-1_{01}$, as detected by Odin towards W Hya. The three thick vertical lines mark where the two correlator bands overlap and end.

the peak of the spectrum corresponds to an effective temperature of $2500 \mathrm{~K}$ (Justtanont et al. 2004), which agrees well with that derived by Haniff et al. (1995). The Hipparcos distance, taken directly from the Hipparcos Catalogue (ESA 1997) parallax, is $115 \mathrm{pc}$. The derived luminosity for this distance turns out to be very high $-1.16 \times 10^{4} L_{\odot}$ (estimated from a fit to the SED). However, the Hipparcos data were reanalysed by Knapp et al. (2003). The revised distance of $78 \mathrm{pc}$, results in a luminosity of $5400 L_{\odot}$, more in line with it being an early-AGB star. The resulting radius is $2.73 \times 10^{13} \mathrm{~cm}$. From this point on, we will assume a distance of $78 \mathrm{pc}$ for our calculations.

\subsection{CO lines}

The code used to calculate the CO line emission is based on the work by Schöier \& Olofsson (2001). It employs the Monte-Carlo method to determine the excitation in the lowest 40 rotational levels in the ground and first excited vibrational states. The collisional rates are taken from the $\mathrm{CO}-\mathrm{H}_{2}$ rates by Flower (2001), and extrapolated to higher rotational levels and temperatures as described in Schöier et al. $(2005)^{1}$. The rates for ortho- and para- $\mathrm{H}_{2}$ collisions are weighted assuming an ortho/para ratio of three. The outer radius is defined by the photodissociation of $\mathrm{CO}$, and it is obtained following the results of Mamon et al. (1988). In order to convert the CO gas mass-loss rate to the total gas mass-loss rate, we assume a $\mathrm{CO}$ abundance, $[\mathrm{CO}] /\left[\mathrm{H}_{2}\right]$, of $2 \times 10^{-4}$.

In the fitting of observed line fluxes of $\mathrm{CO} J=1-0$ up to 4-3, along with the ISO-LWS $J=16-15$ and 17-16 lines, the gas kinetic temperature is calculated with a dust-drag heating

1 The molecular datafile is available for download at www.strw. leidenuniv.nl/ moldata 

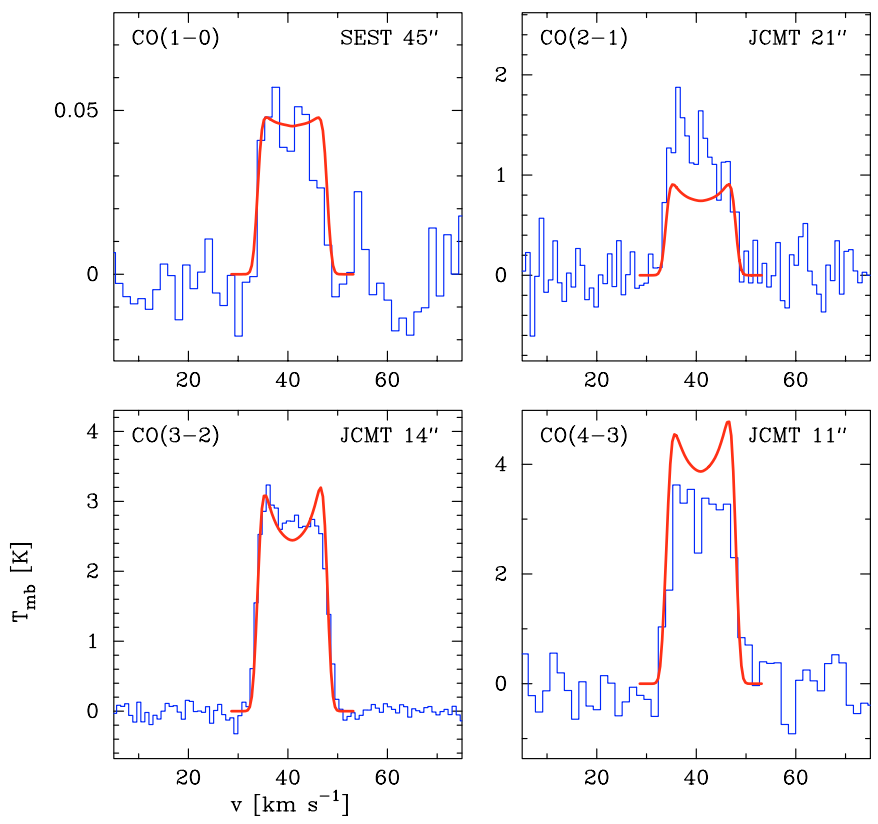

Fig. 2. The calculated best-fit model line profiles for CO $J=1-0,2-1$, $3-2$, and 4-3 (continuous line) and the observed profile (histogram).

balanced by the adiabatic and radiative cooling. The radiative cooling is provided by both $\mathrm{CO}$ and $\mathrm{H}_{2} \mathrm{O}$ molecules. The dust parameters entering in the heating term, due to collisions between dust and gas particles, are taken to be consistent with those obtained from the dust modelling. In the case of a lowmass-loss-rate object such as $\mathrm{W}$ Hya, the $\mathrm{CO}$ molecules are mainly excited by the stellar radiation field and the temperature structure plays only a minor role (Schöier \& Olofsson 2001). A best-fit model is obtained in terms of integrated line intensities and in the $\chi^{2}$ sense,

$\chi^{2}=\sum_{i=1}^{N} \frac{\left(I_{\mathrm{mod}, i}-I_{\mathrm{obs}, i}\right)^{2}}{\sigma_{i}^{2}}$,

where $I$ is the total integrated line intensity, $\sigma_{i}$ is the uncertainty in observation $i$, and the summation is done over all independent observations $N$. The errors in the observed radio line intensities are assumed to be $20 \%$, while the ISO lines have an uncertainty of $30 \%$. The result is a stellar mass-loss rate of $1.3 \times 10^{-7} M_{\odot} \mathrm{yr}^{-1}$.

Although our calculated line fluxes are close to the observed, the line profiles do not match. We consistently expect to see double-peaked profiles while the observations tell us otherwise. A way to remedy this is to decrease the envelope size and increase the input mass-loss rate. A mass-loss rate of $1.3 \times 10^{-7} M_{\odot} \mathrm{yr}^{-1}$ results in a $\mathrm{CO}$ photodissociation radius of $1.4 \times 10^{16} \mathrm{~cm}$ according to Mamon et al. (1988). However, our best-fit model, taking into account also the line shapes, has an outer $\mathrm{CO}$ radius of $8 \times 10^{15} \mathrm{~cm}$ with a mass-loss rate of $2 \times 10^{-7} M_{\odot} \mathrm{yr}^{-1}$. The results of the best-fit model are shown in Fig. 2 and the line fluxes are listed in Table 1. The uncertainty in the mass-loss rate from fitting the line profile is of the order of $50 \%$ within the adopted circumstellar model (see Schöier $\&$ Olofsson 2001). The final model also gives the LSR velocity for W Hya as $40.7 \mathrm{~km} \mathrm{~s}^{-1}$, and an envelope gas expansion

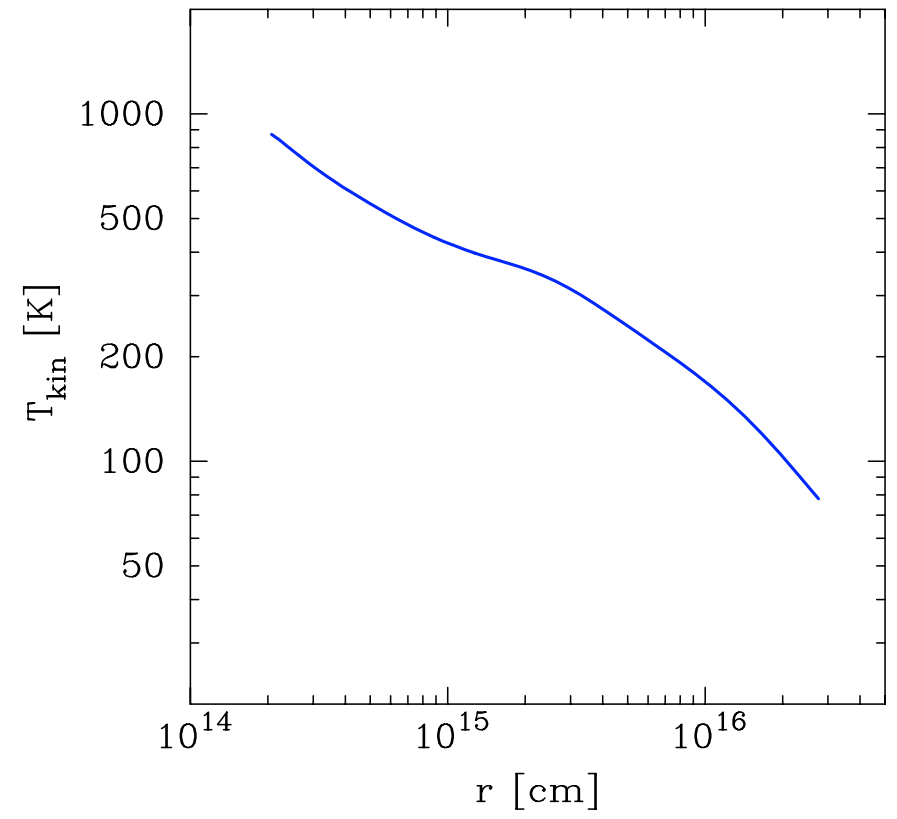

Fig. 3. Kinetic temperature profile for the circumstellar envelope of W Hya derived from our best-fit CO model.

Table 1. Modelled line fluxes for CO compared with the observations (the unit is $\mathrm{K} \mathrm{km} \mathrm{s}^{-1}$, except for the ISO lines where the unit is $\mathrm{W} \mathrm{cm}{ }^{-2}$ ). The observed data are taken from the following references: a - Kerschbaum \& Olofsson (1999), b - JCMT archive, c - Cernicharo et al. (1997), d - Olofsson, private communication, e - Young (1995), f - Barlow et al. (1996).

\begin{tabular}{clccc}
\hline \hline Transition & Instr. & Observed flux & Model flux & Ref. \\
\hline $1-0$ & SEST & 0.82 & 0.66 & $\mathrm{a}$ \\
$2-1$ & JCMT & 18.7 & 11.8 & $\mathrm{~b}$ \\
$2-1$ & IRAM & 22.0 & 26.2 & $\mathrm{c}$ \\
$3-2$ & JCMT & 40.2 & 39.8 & $\mathrm{~d}$ \\
$3-2$ & CSO & 29.0 & 22.7 & $\mathrm{e}$ \\
$4-3$ & JCMT & 44.6 & 60.8 & $\mathrm{~b}$ \\
$4-3$ & CSO & 40.0 & 34.8 & $\mathrm{e}$ \\
$16-15$ & LWS & $3.3 \times 10^{-20}$ & $2.4 \times 10^{-20}$ & $\mathrm{f}$ \\
$17-16$ & LWS & $2.3 \times 10^{-20}$ & $2.4 \times 10^{-20}$ & $\mathrm{f}$ \\
\hline
\end{tabular}

velocity of $7.0 \mathrm{~km} \mathrm{~s}^{-1}$. These values are consistent with those derived from the Odin $\mathrm{H}_{2} \mathrm{O}$ data.

The final kinetic temperature profile is shown in Fig. 3. We note that our derived temperature is between 100-150 K higher than that obtained by Zubko \& Elitzur (2000) in the region where the $\mathrm{H}_{2} \mathrm{O}$ molecules are located $\left(r \lesssim 2 \times 10^{15} \mathrm{~cm}\right)$. This is not inconsistent considering our lower mass-loss rate.

\section{2. $\mathrm{H}_{2} \mathrm{O}$ lines}

We model the water lines observed by Odin, ISO-SWS (Neufeld et al. 1996), and ISO-LWS (Barlow et al. 1996). The code used for this differs from the code used for CO. The main reason for a different treatment is the extremely high optical depths in the water lines preventing the convergence of the 
Table 2. Resulting ortho- $\mathrm{H}_{2} \mathrm{O}$ line fluxes for the best-fit model with a stellar mass-loss rate of $2 \times 10^{-7} M_{\odot} \mathrm{yr}^{-1}$ and an ortho- $\mathrm{H}_{2} \mathrm{O}$ abundance of $1.1 \times 10^{-3}$. The lines listed are the Odin line $(538.29 \mu \mathrm{m})$ and some ISO lines previously detected by ${ }^{a}$ Barlow et al. (1996) and ${ }^{b}$ Neufeld et al. (1996). See text for details on the observed line fluxes and their uncertainties.

\begin{tabular}{lccc}
\hline \hline$\lambda(\mu \mathrm{m})$ & Instr. & \multicolumn{2}{c}{ Line flux $\left(10^{-20} \mathrm{~W} \mathrm{~cm}^{-2}\right)$} \\
\hline & & Obs & Mod \\
\hline 538.29 & Odin & $0.36 \pm 0.1$ & 0.45 \\
$179.53^{a}$ & LWS & $10.3 \pm 1.7$ & 11.6 \\
$174.63^{a}$ & LWS & $11.9 \pm 1.2$ & 8.33 \\
$108.07^{a}$ & LWS & $22.8 \pm 4.0$ & 15.3 \\
$66.44^{a}$ & LWS & $32.7 \pm 12.1$ & 21.5 \\
$40.69^{b}$ & SWS & $23.4 \pm 5.3$ & 32.6 \\
$31.77^{b}$ & SWS & $36.3 \pm 3.7$ & 31.1 \\
\hline
\end{tabular}

Monte-Carlo calculation. The technique employed here is the accelerated lambda iteration (Rybicki \& Hummer 1991), which is used to calculate the excitation in the 45 lowest rotational levels of ortho- and para- $\mathrm{H}_{2} \mathrm{O}$.

We use the collisional rates calculated for $\mathrm{H}_{2} \mathrm{O}$ and $\mathrm{He}$ (Green et al. 1993), with a scaling factor of 1.4 to correct for the mass difference between $\mathrm{H}_{2}$ and He. However, a more recent calculation for the collision between $\mathrm{H}_{2} \mathrm{O}$ and ortho- and para$\mathrm{H}_{2}$ by Phillips et al. (1996) suggests that adopting the collisional rate with $\mathrm{He}$ and using the simple scaling factor can give very different rates. Unfortunately, the collisional rates with $\mathrm{H}_{2}$ were calculated up to $140 \mathrm{~K}$, while the temperature in the circumstellar envelope of an AGB star can be up to $1000 \mathrm{~K}$. If the $\mathrm{H}_{2} \mathrm{O}-\mathrm{He}$ rate is underestimated by a large factor, it may have a repercussion on the calculated line fluxes as the water lines are sub-thermally excited in the envelope of W Hya.

The envelope gas expansion velocity, and the kinetic temperature law used are those obtained from the CO line modelling. The outer radius of the water molecules is taken from the measurements of the OH masers by Szymczak et al. (1998), who give an outer $\mathrm{OH}$ shell radius of $1.3^{\prime \prime}$, i.e., $1.5 \times 10^{15} \mathrm{~cm}$ at the assumed distance.

The mass-loss rate and the ortho- $\mathrm{H}_{2} \mathrm{O}$ abundance are free parameters in the fitting procedure where the integrated line intensities of the Odin and ISO lines are used as observational constraints to find the best-fit model in the $\chi^{2}$ sense. The observed integrated line intensities and their uncertainties are given in Table 2. We obtained ISO fluxes by fitting a Gaussian to archived data with the latest calibration file (OLP version 10), rather than using the line fluxes from Barlow et al. (1996) and Neufeld et al. (1996). The error in the observed flux reflects only the estimation of the baseline and does not include possible systematic error or that of the absolute flux.

The results of the line emission modelling are given in Fig. 4 in terms of the minimum $\chi^{2}$ for a given combination of ortho- $\mathrm{H}_{2} \mathrm{O}$ abundance and mass-loss rate. Clearly, the circumstellar $\mathrm{H}_{2} \mathrm{O}$ line emission suggests a relatively low massloss rate. Using a $1 \sigma$ uncertainty we infer a mass-loss rate of $(2.5 \pm 0.5) \times 10^{-7} M_{\odot} \mathrm{yr}^{-1}$, and an ortho- $\mathrm{H}_{2} \mathrm{O}$ abundance

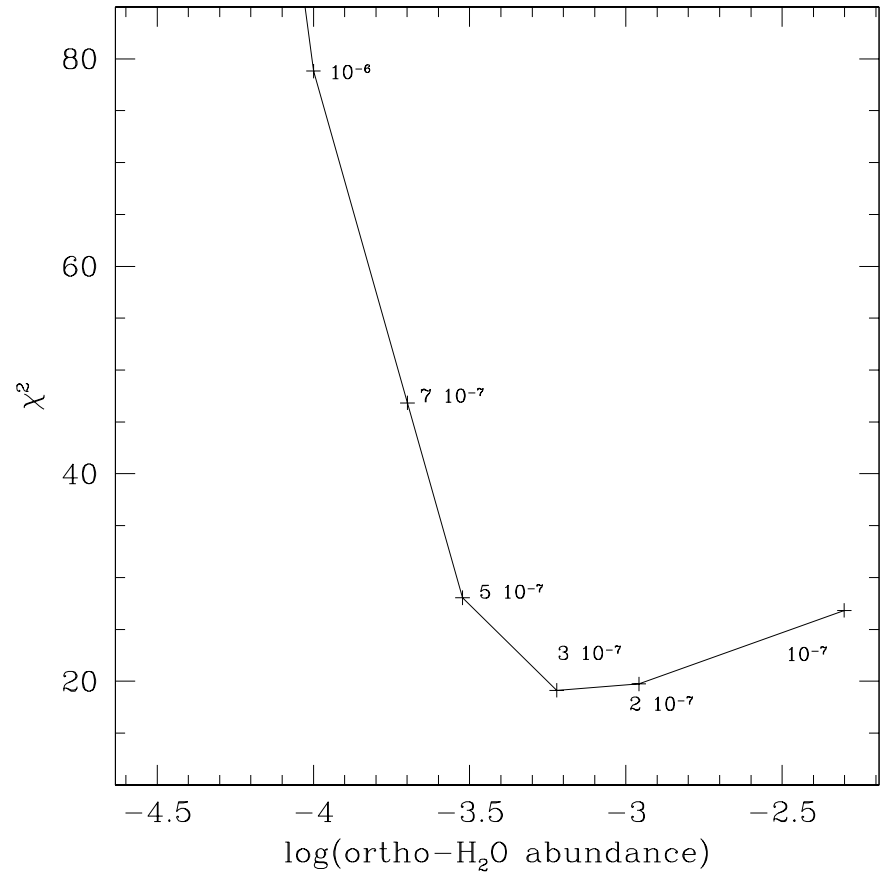

Fig. 4. The minimum $\chi^{2}$ for a given combination of ortho- $\mathrm{H}_{2} \mathrm{O}$ abundance and stellar mass-loss rate (the number at each point). The Odin and ISO LWS/SWS water line fluxes have been used as observational constraints (see Table 2).

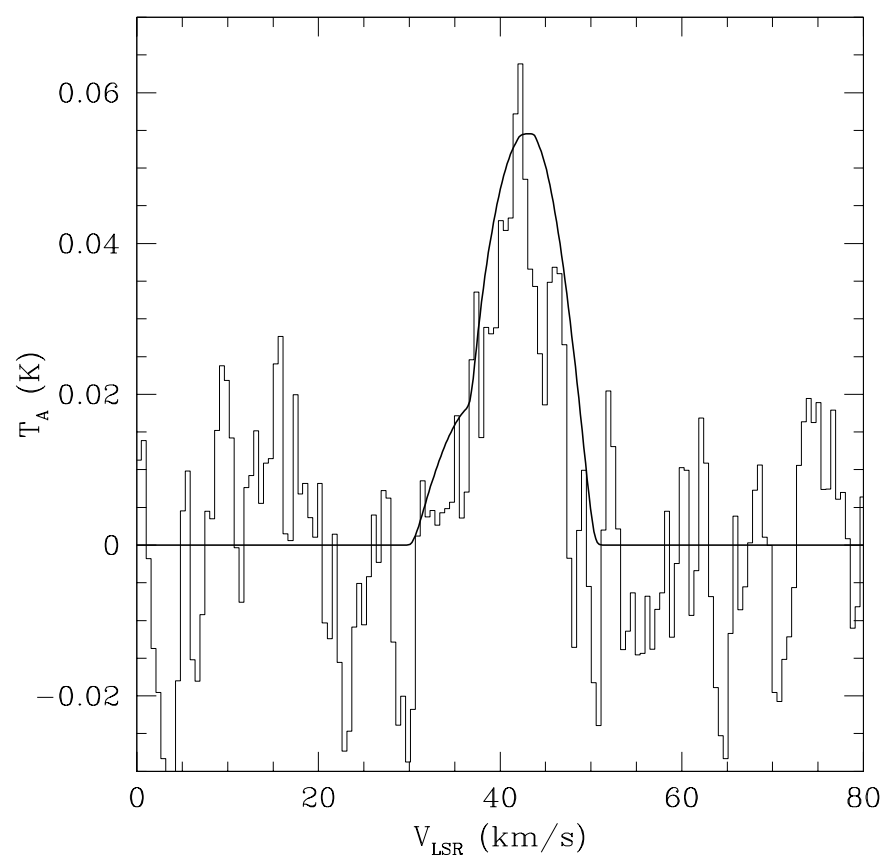

Fig. 5. A comparison between the Odin $\mathrm{H}_{2} \mathrm{O} 1_{10}-1_{01}$ observations (histogram) and the model profile from the best-fit model. The model profile shows clearly the self-absorbed blue wing.

of $(1.0 \pm 0.4) \times 10^{-3}$. The best-fit model line profile for the Odin $557 \mathrm{GHz}$ line is presented in Fig. 5, superimposed on the observed spectrum. The resulting Odin and ISO lines are shown in Fig. 6 although at a much higher spectral resolution than achieved by ISO. 


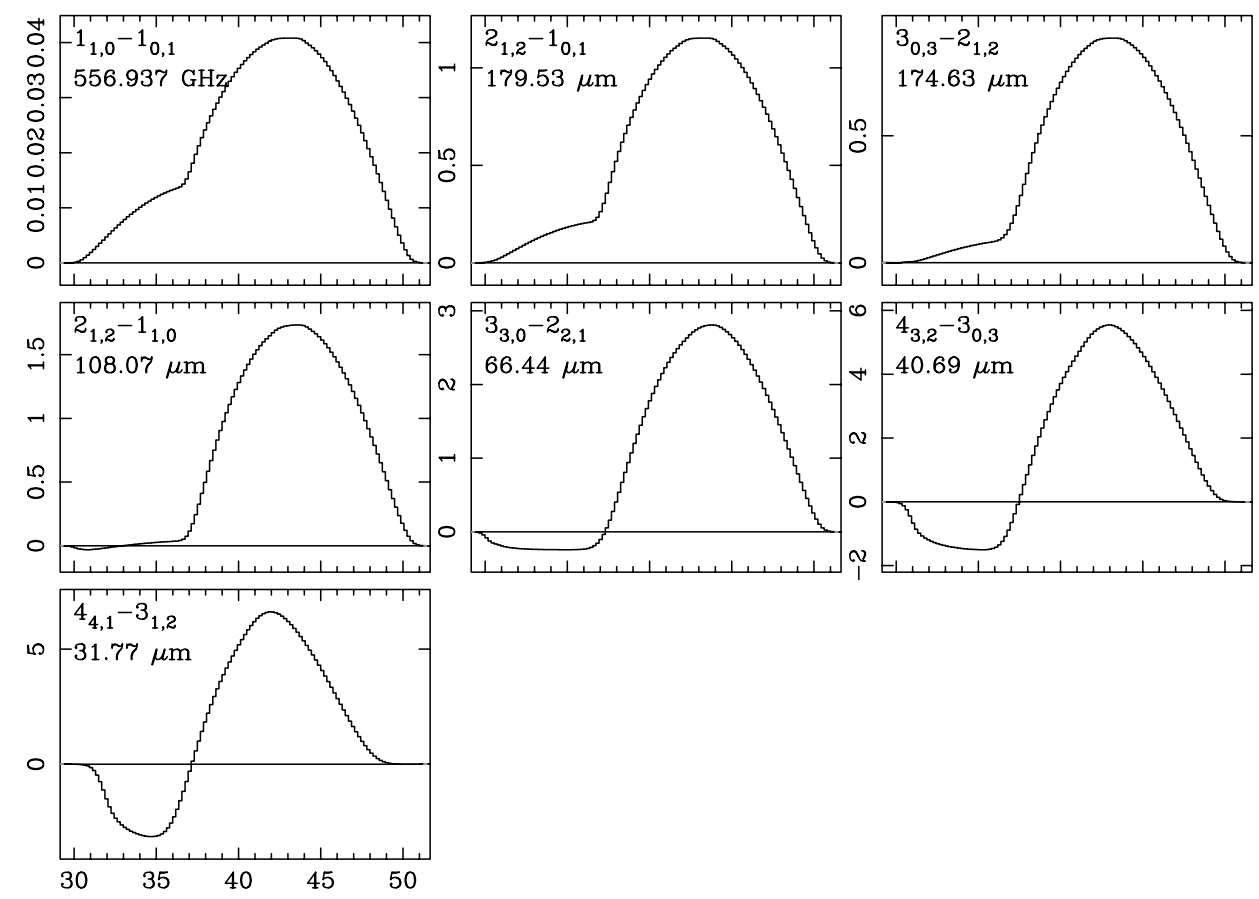

Fig. 6. The calculated line profiles for ortho-water from the best-fit model. The $x$-axis is velocity in $\mathrm{km} \mathrm{s}^{-1}$ and the $y$-axis is flux in the unit of $10^{-20} \mathrm{~W} \mathrm{~cm}^{-2}\left(\mathrm{~km} \mathrm{~s}^{-1}\right)^{-1}$. The numbers in each box indicate the transition and the corresponding wavelength.

We have also modelled the para-water lines present in the ISO spectrum. Since the ortho- and para-water molecules do not interact with each other, they can be modelled independently. In order to fit these lines for the mass-loss rate derived above, the required para-water abundance is $1.1 \times 10^{-3}$ (with an estimated uncertainty similar to that of the ortho$\mathrm{H}_{2} \mathrm{O}$ abundance), The ortho-to-para ratio is close to unity, in agreement with that reported by Barlow et al. (1996). This ratio is expected to be three when considering newly formed $\mathrm{H}_{2}$ on the grain surface at high temperature (Tielens \& Allamandola 1987). The ratio drops to zero for a cold gas.

The total (ortho + para) water abundance according to this analysis, consistent with the observed intensities, is therefore $(2.0 \pm 1.0) \times 10^{-3}$.

The marked increase in the $\chi^{2}$ value for higher mass-loss rates is primarily an effect of the increasing difficulty to fit the SWS lines. The model line fluxes are significantly lower than the observed ones due to the fact that the blue edge of the profile is in absorption, cancelling out the emission from the red side of the profile (see Fig. 6). The absorption is not so pronounced in the low-mass-loss-rate model, hence the overall line fluxes agree more with the observations. It is unfortunate that the resolution of the ISO lines is not high enough for the lines to be resolved. It should be remarked here that the SWS lines come from the very inner region of the circumstellar envelope, and our modelling of these lines is more uncertain. Fitting only the LWS lines gives a weaker constraint on the combination of the mass-loss rate and water abundance.

We have also investigated the effect of the assumed radius of the water envelope by varying this with respect to that adopted from the $\mathrm{OH}$ maser data. By increasing the radius by a factor of three, the resulting line fluxes changed by less than
$50 \%$ for our best-fit model. The line fluxes are primarily excitation limited and they depend only weakly on the adopted $\mathrm{H}_{2} \mathrm{O}$ envelope size. Netzer \& Knapp (1987) derived a size of the water envelope from a theoretical model of $\mathrm{H}_{2} \mathrm{O}$ photodissociation

$R_{\text {out }}=110^{15}\left(\dot{M} / 10^{-7}\right)^{0.7} \mathrm{~cm}$,

where the mass-loss rate is given in $M_{\odot} \mathrm{yr}^{-1}$. We find that the radius obtained from the $\mathrm{OH}$ maser data is fully consistent with this relation for the mass-loss rate of $2.5 \times 10^{-7} M_{\odot} \mathrm{yr}^{-1} \mathrm{ob}-$ tained from the $\mathrm{H}_{2} \mathrm{O}$ line flux fitting.

In our calculation, we did not include the effect of radiative excitation through the vibrational lines. W Hya has a deep $\mathrm{H}_{2} \mathrm{O}$ absorption band at $3 \mu \mathrm{m}$, due to the stretching mode, as seen in the ISO SWS spectrum (Justtanont et al. 2004). This band occurs close to the stellar photosphere $\left(T_{\mathrm{ex}} \sim 10^{3} \mathrm{~K}\right)$ and most likely a significant fraction of the stellar flux at the wavelengths of the vibrational lines is absorbed in it (Ryde et al. 2002). The detection of $\mathrm{H}_{2} \mathrm{O}$ maser emission from rotational lines in the $v_{2}$ vibrational state towards $\mathrm{W}$ Hya shows that radiative excitation to this state occurs (Menten \& Melnick 1989). Deguchi \& Rieu (1990) found that vibrational radiative excitation plays a minor role when calculating the rotational line fluxes in the ground state. On the contrary, Troung-Bach et al. (1999), in their study of the O-rich Mira variable R Cas, found that the ground-state line fluxes decreased by about a factor of two when radiative excitation through the $v_{2}$ vibrational state was removed. We believe that the uncertainty in the $\mathrm{H}_{2} \mathrm{O}$ abundance introduced by omitting radiative excitation through vibrational states is less than our estimated uncertainty from the line modelling. The effect is likely to lower the abundance somewhat. We note here that Truong-Bach et al. derive an $\mathrm{H}_{2} \mathrm{O}$ abundance for R Cas which is remarkably low (in terms of our modelling 
results), $1 \times 10^{-5}$, considering that $\mathrm{R}$ Cas and $\mathrm{W}$ Hya have very similar mass-loss rates, luminosity, distances, and ISO line fluxes.

\section{The mass-loss rate of W Hya}

There is a large discrepancy when it comes to the determination of the mass-loss rate of $\mathrm{W}$ Hya as a wide range (two orders of magnitudes) of the mass-loss rate has been reported over the years. In this paper we have shown that the circumstellar CO radio lines suggest a low mass-loss rate, about $2 \times 10^{-7} M_{\odot} \mathrm{yr}^{-1}$. This result has a rather low uncertainty provided that the adopted circumstellar model and CO abundance $\left(2 \times 10^{-4}\right)$ are correct. Our result is also consistent with those of Wannier \& Sahai (1986), Young (1995), and Olofsson et al. (2002). Once these values are scaled to the same distance, velocity, and $\mathrm{CO}$ abundance, the range of the mass-loss rates is (5-20) $\times 10^{-8} M_{\odot} \mathrm{yr}^{-1}$.

Previous reports on the mass-loss rates derived from $\mathrm{H}_{2} \mathrm{O}$ line emission are based on ISO results. The mass-loss rate estimates range from as high as $10^{-5}$ (assumed distance $95 \mathrm{pc}$ and SWS lines; Neufeld et al. 1996) to $6 \times 10^{-7} M_{\odot} \mathrm{yr}^{-1}$ (assumed distance $130 \mathrm{pc}$ and LWS lines; Barlow et al. 1996). Barlow et al. used a water abundance of $8 \times 10^{-4}$ for $r \leq 6 \times 10^{14} \mathrm{~cm}$ and $3 \times 10^{-4}$ beyond this radius. Neufeld et al. do not give their assumed water abundance, but it must have been low, according to our results (see Fig. 4). We find that the Odin and ISO $\mathrm{H}_{2} \mathrm{O}$ line intensities suggest a mass-loss rate of $2.5 \times 10^{-7} M_{\odot} \mathrm{yr}^{-1}$, a value fully consistent with our $\mathrm{CO}$ line modelling result.

The $\mathrm{OH}$ maser line data give some hints on the massloss rate. Recently, the $1612 \mathrm{MHz}$ line was detected (Etoka et al. 2003), and it is substantially weaker than the main lines at 1665 and $1667 \mathrm{MHz}$. This is an indication of a low massloss rate (Szymczak \& Engels 1998). Likewise, as pointed out above, the measured size of the $\mathrm{OH}$ maser envelope is consistent with a low mass-loss rate.

Justtanont et al. (2004) derived a low dust mass-loss rate by fitting the SED. Using their derived dust mass-loss rate combined with a dynamical calculation of a dust-driven wind, we infer a total mass-loss rate of $1.4 \times 10^{-7} M_{\odot} \mathrm{yr}^{-1}$. This value is only a lower limit because the different dust components used in the SED modelling do not account for the $13 \mu \mathrm{m}$ dust feature. Once the carrier is found for this feature and taken into account in the model, it is possible that the dust mass loss rate can increase by up to a factor of two. The indication from the dynamical calculation is W Hya has a relatively low mass-loss rate.

However, Zubko \& Elitzur (2000) reported a rather high mass-loss rate when combining SED modelling and a dynamical model for the outflow, $2.3 \times 10^{-6} M_{\odot} \mathrm{yr}^{-1}$ (at a distance of $115 \mathrm{pc}$ ). The high mass-loss rate is because the authors did not fit the $10 \mu \mathrm{m}$ silicate feature which is the key in deriving the dust mass loss rate.

Based on these results we conclude that there is strong evidence that $\mathrm{W}$ Hya has a rather low mass-loss rate, of the order a few $10^{-7} M_{\odot} \mathrm{yr}^{-1}$.

\section{The circumstellar $\mathrm{H}_{2} \mathrm{O}$ abundance}

In this work, we derive a consistent mass-loss rate from both the $\mathrm{CO}$ and $\mathrm{H}_{2} \mathrm{O}$ modelling of $(2.5 \pm 0.5) \times 10^{-7} M_{\odot} \mathrm{yr}^{-1}$. The observed line fluxes are reasonably well produced. Our derived water abundance for this mass-loss rate is exceptionally high, $(2.0 \pm 1.0) \times 10^{-3}$. Additional uncertainties in this estimate are provided by the effects of radiative excitation through vibrational states, and the uncertain values for the collisional cross sections.

This derived total water abundance violates the limit set by the cosmic abundance of atomic oxygen. In the stellar equilibrium chemistry of an oxygen-rich environment, all the available carbon should be locked up in the form of $\mathrm{CO}$, and the rest of the oxygen goes into the formation of $\mathrm{H}_{2} \mathrm{O}$ molecules, with a very small fraction bound into grains and other molecules. The cosmic abundance of carbon and oxygen relative to $\mathrm{H}$ is $3.6 \times 10^{-4}$ and $8.5 \times 10^{-4}$, respectively (Anders $\&$ Grevesse 1989). Therefore, unless there is a separate influx of water from another source the water abundance relative to $\mathrm{H}_{2}, f_{\mathrm{H}_{2} \mathrm{O}}=\left[\mathrm{H}_{2} \mathrm{O}\right] /\left[\mathrm{H}_{2}\right]$, cannot exceed $10^{-3}$.

The extra water mass required to explain the abundance needed to model the observed water line emission is

$M_{\mathrm{H}_{2} \mathrm{O}}=\dot{M}\left(f_{\mathrm{H}_{2} \mathrm{O}}-10^{-3}\right)\left(r_{\mathrm{H}_{2} \mathrm{O}} / v\right)\left(m_{\mathrm{H}_{2} \mathrm{O}} / m_{\mathrm{H}_{2}}\right)$

where $r_{\mathrm{H}_{2} \mathrm{O}}$ is the radius of the water shell, and $v$ the gas expansion velocity. The total extra mass needed is $(6.1 \pm 3.0) \times 10^{26} \mathrm{~g}$, i.e., $(0.1 \pm 0.05) M_{\oplus}$. This is considerably smaller than the estimated mass of water ice in the Solar System, assuming most of the present mass of water is bound in comets. The current estimate of the original mass of the Oort Cloud is highly uncertain and varies between 14-1000 $M_{\oplus}$ (Bailey \& Stagg 1988; Weissman 1991; Marochnik et al. 1988). We speculate that icy bodies can be either part of the disk (and planets) left over after the formation of the central star, or a remnant envelope of the original cloud from which the star formed, reminiscent of the Oort Cloud. The disk/envelope remained in stable orbits until the star ascended the AGB. Because of the much higher luminosity of the AGB star, compared to that in its main sequence phase, outgassing from icy bodies will contribute to the observed high water abundance. Of course, this requires a steady supply of new water, since photodissociation effectively destroys the water molecules on a short time scale. A similar hypothesis has been put forward to explain the presence of gaseous water around the carbon star IRC+10216 (Melnick et al. 2001).

\section{Summary}

Observations of the ground-state transition of ortho-water, $1_{10}-1_{01}$, in the circumstellar envelope of $\mathrm{W}$ Hya were done using the Odin satellite. The line is centered at $V_{\mathrm{LSR}}=42 \mathrm{~km} \mathrm{~s}^{-1}$ and its width corresponds to a gas expansion velocity of about $7 \mathrm{~km} \mathrm{~s}^{-1}$. These results compare well with those of previous CO observations (e.g., Kerschbaum \& Olofsson 1999), as well as the new CO data presented in this paper. The line flux is $(3.6 \pm 1.2) \times 10^{-21} \mathrm{~W} \mathrm{~cm}^{-2}$, consistent with the SWAS observation of the same line (Harwit \& Bergin 2002). 
To fit the Odin and ISO water lines, the ortho-water abundance and mass-loss rate are required to be $(1.0 \pm 0.4) \times 10^{-3}$ and $(2.5 \pm 1.0) \times 10^{-7} M_{\odot} \mathrm{yr}^{-1}$, respectively. Similarly, the parawater abundance is estimated to be $(1.1 \pm 0.8) \times 10^{-3}$ by fitting ISO lines. This mass-loss rate is consistent with that derived from our CO line modelling, as well as that obtained by combining SED modelling and a dynamical model of the outflow. From our calculation, we show that the line shapes of these water transitions are different. Unfortunately, from ISO, we have only the information on the line intensities. It is noted here that the Heterodyne Instrument for the Far-Infrared (HIFI) aboard the Herschel Observatory which will be launched around 2008 will have a much higher resolution which can resolve some of these ISO lines, thereby giving another constraint on the model parameters.

If the mass-loss rate for $\mathrm{W}$ Hya is as low as $(2.5 \pm 0.5) \times 10^{-7} M_{\odot} \mathrm{yr}^{-1}$, the total water abundance needed to explain the observed line fluxes is $(2.0 \pm 1.0) \times 10^{-3}$. However, if we assume the cosmic abundance for carbon and oxygen, the maximum total water abundance, relative to $\mathrm{H}_{2}$, cannot be higher than $10^{-3}$. A way to reconcile the high total water abundance needed to model the $\mathrm{H}_{2} \mathrm{O}$ lines of $\mathrm{W}$ Hya is an extra injection of water in the system, possibly from evaporation of icy bodies, such as planets or comets. This is the suggested explanation for the detection of water in IRC+10216, the IRbrightest carbon-rich AGB star (Melnick et al. 2001). The excess $\mathrm{H}_{2} \mathrm{O}$ mass needed to explain the Odin and ISO observations in W Hya is small, of the order of $(0.1 \pm 0.05) M_{\oplus}$, compared to the estimate of the original mass of the Oort Cloud.

Acknowledgements. This work is partly funded by the Swedish National Space Board. We wish to thank René Liseau and Göran Olofsson for fruitful discussions. Our research made use of the database SIMBAD.

\section{References}

Anders, E., \& Grevesse, N. 1989, Geochem. Cosmochim. Acta, 53, 197

Bailey, M. E., \& Stagg, C. R. 1988, MNRAS, 235, 1

Barlow, M. J., Nguyen-Q-Rieu, Truong-Bach, et al. 1996, A\&A, 315, L241

Cami, J. 2002, Ph.D. Thesis, University of Leiden

Cernicharo, J., Alcolea, J., Baudry, A., \& González-Alfonso, E. 1997, A\&A, 319, 607

Chapman, J. M., Sivagnanam, P., Cohen, R. J., \& Le Squeren, A. M. 1994, MNRAS, 268, 475

Deguchi, T., \& Rieu, N.-Q. 1990, ApJ, 360, L27

ESA 1997, the Hipparcos and Tycho Catalogues, ESA, SP-1200

Etoka, S., Le Squeren, A. M., \& Gerard, E. 2003, A\&A, 403, L51

Flower, D. R. 2001, J. Phys. B.: At. Mol. Opt. Phys., 34, 2731
Frisk, U., Hagström, M., Ala-Laurinaho, J., et al. 2003, A\&A, 402, L27

Gilman, R. C. 1969, ApJ, 155, L185

Green, S., Maluendes, S., \& McLean, A. D. 1993, ApJS, 85, 181

Habing, H. J. 1996, A\&ARv, 7, 97

Haniff, C. A., Scholz, M., \& Tuthill, P. G. 1995, MNRAS, 276, 640

Harwit, M., \& Bergin, E. A. 2002, ApJ, 565, L105

Hawkins, G. W. 2000, A\&A, 229, L5

Hjalmarson, A, Frisk, U., Olberg, M., et al. 2003, A\&A, 402, L39

Iben, Jr., I., \& Renzini, A. 1983 ARA\&A, 21, 271

Justtanont, K., de Jong, T., Tielens, A. G. G. M., Feuchtgruber, H., \& Waters, L. B. F. M. 2004, A\&A, 417, 625

Kerschbaum, F., \& Olofsson, H. 1999, A\&AS, 138, 299

Knapp, G. R., Pourbaix, D., Platais, I., \& Jorissen, A. 2003, A\&A, 403, 993

Lebzelter, T., Kiss, L. L., \& Hinkle, K. H. 2000 A\&A, 361, 167

Loup, C., Forveille, T., Omont, A., \& Paul, J. F. 1993, A\&AS, 99, 291

Mamon, G. A., Glassgold, A. E., \& Huggins, P. J. 1988 ApJ, 328, 797

Marochnik, L. S., Mukhim, L. M., \& Sagdeev, R. Z. 1988, Science, 242, 547

Melnick, G. J., Neufeld, D. A., Ford, K. E. S., Hollenbach, D. J., \& Ashby, M. L. N. 2001, Nature, 412, 160

Menten, K. M., \& Melnick, G. J. 1989, ApJ, 341, L91

Millar, T. J. 2003, in Asymptotic giant branch stars, Astronomy and astrophysics library, ed. H. J. Habing, \& H. Olofsson (New York, Berlin: Springer), 248

Netzer, N., \& Knapp, G. R. 1987, ApJ, 323, 734

Neufeld, D. A., Chen, W., Melnick, G. J., et al. 1996, A\&A, 315, L237

Nordh, H. L., von Schéele, F., Frisk, U., et al. 2003, A\&A, 402, L21

Ohnaka, K. 2004, A\&A, 424, 1011

Olofsson, H. 2003, in Asymptotic giant branch stars, Astronomy and astrophysics library, ed. H. J. Habing \& H. Olofsson (New York, Berlin: Springer), 325

Olofsson, H., González-Delgado, D., Kerschbaum, F., \& Schöier, F. L. 2002, A\&A, 391, 1053

Pardo, J. R., Alcolea, J., Bujarrabal, V., et al. 2004, A\&A, 424, 145

Phillips, T. R., Maluendes, S., \& Green, S. 1996, ApJS, 107, 467

Rybicki, G. B., \& Hummer, D. G. 1991, A\&A, 245, 171

Ryde, N., Lambert, D. L., Richter, M. J., \& Lacy, J. H. 2002, ApJ, 580,447

Schöier, F. L., \& Olofsson, H. 2001, A\&A, 368, 969

Schöier, F. L., van der Tak, F. F. S., van Dishoeck, E. F., \& Black, J. H. 2005, A\&A, 432, 369

Szymczak, M., Cohen, R. J., \& Richards, A. M. S. 1998, MNRAS, 297,1151

Szymczak, M., \& Engels, D. 1995, A\&A, 296, 727

Tielens, A. G. G. M., \& Allamandola, L. J. 1987, In Interstellar Processes, ed. D. J. Hollenbach, \& H. A. Thronson (Dordrecht: Reidel), 397

Treffers, R, \& Cohen, M. 1974, ApJ, 188, 545

Truong-Bach, Sylvester, R. J., Barlow, M. J., et al. 1999, A\&A 345, 925

Wannier, P. G., \& Sahai, R. 1986, ApJ, 311, 335

Weissman, P. R. 1991, Icarus, 89, 190

Young, K. 1995, ApJ, 445, 872

Zubko, V., \& Elitzur, M. 2000, ApJ, 554, L137 